\title{
Etiología de la infección cérvico vaginal en pacientes del Hospital J uárez de México
}

\author{
Rocío Flores-Paz, Biól, ${ }^{(1)}$ Ro berto Rivera-Sánchez, Q FB, ${ }^{(1)}$ Elvia García-Jíménez, Q BP, ${ }^{(1)}$ \\ Myriam Arriaga-A lba, M en C, D ra en C.(1)
}

\begin{abstract}
Flores-Paz R, Rivera-Sánchez $\mathbf{R}$, García-Jiménez E,Arriaga-Alba M. Etiología de la infección cérvico vaginal en pacientes del Hospital Juárez de México. Salud Publica Mex 2003;45 supl 5:S694-S697. El texto completo en inglés de este artículo está disponible en: http://www.insp.mx/salud/index.html

\section{Resumen}

Objetivo. Conocer la etiología de la infección cérvico vaginal, con el fin de establecer un diagnóstico acertado que permita ofrecer a las pacientes el tratamiento más apropiado. Material y métodos. De enero de 1995 a diciembre de 1999 se realizó un estudio bacteriológico a 6811 muestras de exudado cérvico vaginal de pacientes del Hospital Juárez de México, de la Ciudad de México, con edades comprendidas entre los 13 y los 65 años, que referían leucorrea, prurito, hiperemia y dolor abdominal bajo. Resultados La frecuencia de infección por cada germen fue $\mathrm{G}$ vaginalis, $22.65 \%$, Candida spp, $19.13 \%$, C albicans, $7.8 \%$, T vaginalis, $1.5 \%$, Streptococcus del grupo $D, 11.78 \%$, Streptococcus $\beta$ haemolyticus, $4.59 \%$, E coli, $13.46 \%$, Klebsiella ssp, $2.0 \%$, además de otras enterobacterias menos frecuentes como $\mathrm{Cl}$ trobacter spp, Enterobacter spp, Pseudomonas spp, M morganii y P mirabilis. El $2.9 \%$ presentó anaero bios siempre asociados con $\mathrm{G}$ vaginalis. Se aislaron Neisseria spp y N weaveri en $0.15 \%$ de las muestras. La N gonorrhoeae no se encontró en ningún caso. Datos comparativos indican que, tanto Streptococcus hemoliticos como $\mathrm{E}$ coli tuvieron un marcado incremento en los dos últimos años, siendo el de esta última estadísticamente significativo $(p<0.001)$. El método de ji cuadrada se aplicó para la evaluación de los datos. Conclusiones C onsiderando la diversidad de la etiología de la infección se recomienda realizar cultivos de la secreción cérvico vaginal a to das las pacientes con sinto matolo gía sugestiva de ella. El texto completo en inglés de este artículo está disponible en: http://www.insp.mx/salud/index.html
\end{abstract}

Palabras clave: vaginitis; vaginosis bacteriana; México
Flores-Paz R, Rivera-Sánchez $R$,

García-Jiménez E,Arriaga-Alba M.

Etiology of cervicovaginal infection

in Mexican women.

Salud Publica Mex 2003;45 suppl 5:S694-S697.

The English version of this paper

is available at: http://www.insp.mx/salud/index.html

\begin{abstract}
A bstract
O bjective. To identify the etiologic agents of cervicovaginal infection in order to establish an accurate diagnosis and proper treatment. Material and Methods From January 1995 to December 1999, bacteriological studies were done in cervical discharge specimens from 6811 patients aged 13 to 65 years, seen at Hospital Juarez in Mexico City. All patients had leucorrhea, pruritus, hyperemia, and abdominal pain. Statistical significance was assessed using the chisquared test. Results. The frequencies of infectious agents were as follows: G. vaginalis, $22.65 \%$, Candida spp, $19.13 \%$, C. albicans, $7.8 \%$, T.vaginalis, $1.5 \%$, Streptococcus group D, $11.78 \%$, Streptococcus $\beta$ hemolytic, $4.59 \%$, E. coli, $13.46 \%$, and Klebsiella spp, 2.0\%. Less frequent entero bacteria were:Citrobacter spp, Enterobacter spp, Pseudomonas spp, M . morganii, and P. mirabilis. Almost 3\% of patients presented anaero bic species, which were always asso ciated with $\mathrm{G}$. vaginalis. N eisseria spp and $\mathrm{N}$. weaveri were isolated in $0.15 \%$ each; $N$. gonorrhoeae was not isolated in any of the patients. Comparative data showed that Streptococcus $\beta$ hemolytic and E. coli increased markedly in the past two years ( $p<0.001$ for the latter). Conclusions. The diversity of etiologic agents requires performing bacteriological cultures of cervical and vaginal discharge to all symptomatic patients. The English version of this paper is available at: http://www.insp.mx/salud/index.html
\end{abstract}

Key words: vaginitis; bacterial vaginosis; Mexico

(1) Dirección de Investigación y Enseñanza. Hospital Juárez de México, México, D F, México.

Fecha de recibido: 9 de octubre de 2002 - Fecha de aprobado: 23 de abril de 2003 Solicitud de sobretiros:D ra Myriam A rriaga Alba. Hospital Juárez de México. D irección de Investigación y Enseñanza. Avenida Instituto Politécnico N acional No 5160, colonia Magdalena de las Salinas, 07760 México, DF. Correo electrónico: arriaga_alba@ yahoo.com 
a vaginosis bacteriana (VB), la tricomoniasis y la candidiasis son posiblemente los padecimientos ginecológicos más frecuentes. ${ }^{1,2} \mathrm{La}$ VB se caracteriza por la sustitución de los lactobacillos por Gardnerella vaginalis, asociación que se debe con frecuencia a anaerobios, como Bacteroides spp y Mobiluncus spp. Este padecimiento se manifiesta por flujo, comezón, mal olor, pH elevado, olor de aminas en la prueba de $\mathrm{KOH}$. Cerca de $50 \%$ de las pacientes con VB pueden permanecer temporalmente asintomáticas. ${ }^{3}$ Aunque los estudios de VB datan de hace 40 años su conocimiento sigue siendo confuso, debido a que han existido cambios de nomenclatura del principal microrganismo causante, la $G$ vaginalis, a que los criterios clínicos de la enfermedad no son muy específicos y porque este padecimiento continúa siendo subdiagnosticado. La frecuencia de $\mathrm{VB}$ en la población mundial varía entre 16 y $26 \%$.

La vaginitis por Candida spp, caracterizada por ardor, comezón, flujo, inflamación de la vagina, es frecuente en la mujer. El principal agente etiológico de la candidiasis vaginal y de otras infecciones ginecológicas como la peritonitis pélvica es $C$ albicans ${ }^{4,5}$ En población mexicana $60 \%$ de 234 pacientes tuvieron cultivos positivos para C albicans y $40 \%$ para Candida spp. ${ }^{6}$

La frecuencia de candidiasis vaginal difiere en cada población. En Turquía, el principal agente causal de vaginitis fue Escherichia coli, seguido de Candida spp. ${ }^{7}$ En Colombo, Alemania, Perera y Clayton ${ }^{4}$ encontraron que en $32 \%$ de las pacientes con vaginitis ésta era debida a Candidas, y en la India, 20\% tenían Candida. ${ }^{8}$ La frecuencia de infecciones por Candida no albicans se ha incrementado en las últimas dos décadas en los Estados Unidos de América (EUA); entre éstas las más importantes parecen ser $C$ tropicales y $C$ glabrata, por abuso de medicamentos. ${ }^{9}$

Otro agente etiológico causante de vaginitis es el parásito Trichomona vaginalis, que causa lesiones muy severas en el cérvix como friabilidad, zonas hemorrágicas y secreción vaginal abundante. Su frecuencia es muy variada; dependiendo de la población estudiada varía por ejemplo desde $1.7 \%$, en Cuernavaca, México, $2.5 \%$ en la Unión Soviética, $14 \%$ en Mauritania, hasta $35 \%$ en Haití. ${ }^{10-13}$

Esta diversidad de resultados, en los estudios epidemiológicos de la infección cérvico vaginal, enriquecen la evidencia de que la etiología de esta infección es variable y requiere la realización de estudios individuales para conocer las características de la población con la que se esté trabajando.

\section{Material y métodos}

Durante 1995-1999 se tomaron muestras de exudado cérvico vaginal a 6811 pacientes, entre los 13 y 65 años de edad. Las pacientes fueron enviadas al Laboratorio de Investigación Microbiológica, previa evaluación por los Servicios de Biología de la Reproducción Humana o de Ginecología, del Hospital Juárez de México. Todas las pacientes referían cursar con cuadros de infecciones recurrentes, leucorrea, prurito, hiperemia y dolor abdominal bajo. Se excluyeron de este trabajo aquellas pacientes con tratamientos por vía vaginal, y/o sangrado o con relaciones sexuales recientes.

Se les aplicó un cuestionario acerca de la sintomatología que presentaban.Posteriormente, se les introdujo un espejo vaginal estéril para observar la presencia o no de flujo y sangrado. Se utilizaron tres hisopos en cada paciente: con el primero se tomó muestra del orificio del cérvix y se colocó en medio de Thayer-Martin, medición de $\mathrm{pH}, \mathrm{KOH}$ (a 10\%), para determinación de presencia de aminas; con el segundo, se tomó muestra de la secreción del fondo de saco y se colocó en un medio de transporte como caldo soya tripticaseina, y con el tercero se toma muestra de fondo de saco y se coloca en solución salina isotónica para efectuar el análisis microscópico en fresco.

Todas las muestras se analizaron con base en los criterios de Amsel (medición de $\mathrm{KOH}, p H>5.0$ ), examen microscópico en fresco para observar células clave, levaduras, T vaginalis y leucocitos) tinción de Gram y cultivos bacteriológicos en medio de gelosa sangre, agar chocolate, agar de Mc Conckey, agar de Thayer Martin, medio HBT de doble capa, con sangre humana y agar de Biggy. Todos los cultivos bacteriológicos se hicieron de acuerdo con los métodos descritos por Insenberg y D' Amato en 1991. ${ }^{14}$ Todas las colonias aisladas en dichos cultivos bacteriológicos fueron identificadas con pruebas bioquímicas previamente establecidas, empleando el método semi-automatizado API20E de Biomeriux para la identificación de enterobacterias, pruebas de hipurato e hidrólisis de almidón, para la identificación de $G$ vaginalis, y los métodos descritos por Insenberg y D' Amato para la identificación de microrganismos Gram positivos. Las Candidas aisladas en medio de Biggy fueron identificadas mediante la formación de tubo germinativo en suero humano. ${ }^{15}$

El incremento de especies bacterianas durante el periodo estudiado se analizó empleando la prueba de ji cuadrada. 


\section{Resultados}

En este grupo de 6811 pacientes la infección vaginal fue identificada como VB en $22.65 \%$ de los casos; cerca de $3 \%$ tuvo infección mixta, asociada con anaerobios como Mobiluncus spp. El cuadro I muestra un resumen de 31 diferentes especies de microrganismos identificados en los 6811 cultivos vaginales estudiados entre 1995 y 1999. Las especies causantes de vaginitis identificadas fueron T vaginales, C Albicans, Candida spp, 19 especies diferentes de Gram negativos y ocho de Gram positivos (cuadro I). En el cuadro II se observa

Cuadro I

\section{Microrganismos IDentificados en mUestras de EXUDADO CÉRVICO VAGINAL EN PACIENTES CON infeCción SintomÁtica. CiUdad de MéXico, 1995- 1998. MÉXICO}

\begin{tabular}{|c|c|c|}
\hline Especie & Número & $(\%)$ \\
\hline E coli & 917 & 13.46 \\
\hline K ozaneae & 1 & 0.15 \\
\hline K pneumoniae & 114 & 1.67 \\
\hline K oxytoca & 22 & 0.32 \\
\hline $\mathrm{P}$ mirabilis & 73 & 1.07 \\
\hline$\overline{P \text { penneri }}$ & 1 & 0.015 \\
\hline A calcoaceticus & 1 & 0.015 \\
\hline A haemolyticus & 5 & 0.07 \\
\hline E aerogenes & 5 & 0.07 \\
\hline E agglomerans & 7 & 0.10 \\
\hline E cloacae & 15 & 0.22 \\
\hline E gergoviae & 1 & 0.015 \\
\hline S marscencens & 1 & 0.015 \\
\hline S odorifera & 7 & 0.10 \\
\hline $\mathrm{P}$ aeruginosa & 3 & 0.044 \\
\hline Citrobacter spp & 2 & 0.029 \\
\hline M oraxella spp & 1 & 0.015 \\
\hline M obiluncus spp & 171 & 2.50 \\
\hline$\overline{\mathrm{M} \text { morganii }}$ & 8 & 0.12 \\
\hline Lactobacillus spp & 4969 & 72.96 \\
\hline Corynebacterium spp & 2443 & 26.70 \\
\hline $\mathrm{S}$ aureus & 82 & 1.20 \\
\hline Staphylococcus spp & 1839 & 27.00 \\
\hline Micrococcus spp & 170 & 2.49 \\
\hline S grupo D enterococo & 712 & 10.45 \\
\hline S grupo D no enterococo & 97 & 1.42 \\
\hline S agalactiae & 313 & 4.59 \\
\hline S grupo viridans & 119 & 1.75 \\
\hline Candidas (totales) & 1293 & 19.13 \\
\hline C albicans & 61 & 0.89 \\
\hline $\mathrm{G}$ vaginalis & 1543 & 22.65 \\
\hline $\bar{T}$ vaginalis & 108 & 1.59 \\
\hline
\end{tabular}

la variación de las principales especies identificadas durante el estudio, de lo cual destaca que E coli y Streptococcus del grupo D incrementaron su frecuencia en forma estadísticamente significativa.

\section{Discusión}

La tasa de $G$ vaginalis ( $22.65 \%$ ) fue menor que en otros países de América Latina, como Haití (60\%). ${ }^{13}$ La VB en $2.5 \%$ de los casos se asoció a Mobiluncus spp y a la ausencia de Lactobacillus spp. A la exploración clínica es difícil distinguir VB de vaginitis causada por otros agentes etiológicos. La mejor manera de obtener un diagnóstico confiable de VB es la observación microscópica de células guía junto con el cultivo en medio de dos capas HBT, con sangre humana.

La Candida spp es importante en la patología de la población mexicana. Se encontró que sólo 7.8 \% de 1303 levaduras fueron $C$ albicans. Estos resultados son muy similares a los reportados para otra muestra de población mexicana, en la cual se caracteriza a la Candida como una de las principales causas de vaginitis. ${ }^{10}$ Se observa la conveniencia de identificar las especies de Candida, ya que $C$ glabrata puede causar infecciones recurrentes. ${ }^{16}$

La $T$ vaginalis se observó por examen microscópico sólo en $1.59 \%$ de las muestras. Esta frecuencia es 
moderadamente menor a la reportada en otro grupo de población de la ciudad de Cuernavaca $(2.5 \%),{ }_{1}^{10}$ lo que sugiere que este agente etiológico de vaginitis no es una causa muy frecuente de infección cérvico vaginal en el país.

La Neisseria gonorrhoeae no fue encontrada en ninguna de las 6811 pacientes estudiadas. Pliuto, ${ }^{11}$ en Rusia, reporta $0.27 \%$ en 5787 pacientes de población abierta. Estos datos no son sugestivos de que se haya erradicado este microrganismo en la población mexicana, sino que la población estudiada en el Hospital Juárez de México no lo es para establecer el riesgo de contagio venéreo.

El aislamiento de E coli en vaginitis sintomáticas podría ser significativo, pues se observa un incremento del mismo con el tiempo (11.115\% / 2440 entre 19951996, 16.67\% / 4371 entre 1997-1999 $p>0.001)$. Las enterobacterias no son consideradas como patógenos de la vagina, sin embargo, se observan cultivos con escasos a moderados lactobacilos en pacientes con sintomatología de infección cérvico vaginal y con cultivos de $E$ coli o alguna otra especie de enterobacteria (cuadro I). Estos datos sugieren que es aconsejable conocer mejor la importancia del aislamiento de $E$ coli y sus factores de patogenicidad en vagina y cérvix.

Entre los microrganismos Gram positivos aislados el Streptococcus spp grupo D y S. agalactiae son frecuentes en pacientes con sintomatología, y la mayoría de las veces estos agentes se aislaron en forma de cultivo puro. En la población mexicana estos microrganismos tienen cada vez más importancia, ya que como puede observarse en el cuadro II su frecuencia se ha incrementado en forma considerable. Estos resultados concuerdan con otros reportes, en los que se muestra que $S$ agalactiae debe considerarse como una causa importante de vaginitis. ${ }^{17}$

En conclusión, los microrganismos que se encuentran asociados a vaginitis son numerosos; con los resultados obtenidos en este trabajo se recomienda que el estudio de la infección cérvico vaginal debe hacerse con cuidado, asociando los datos clínicos con los hallazgos bacteriológicos del cultivo de la secreción. El cultivo de la secreción cérvico vaginal es una alternativa certera y aconsejable para establecer un buen diagnóstico de la etiología de la infección en esta región corporal en pacientes sintomáticas.

\section{A gradecimientos}

Se agradece a la señora Gabriela Aguilera Hernández, técnica laboratorista, su apoyo en el trabajo del laboratorio.

\section{Referencias}

1. Hay PE,Taylor-Robinson D. D efining bacterial vaginosis.To BV or not to $B V$, that is the question. Int J ATD-AIDS 1996;7(4):233-235.

2. Thorsen $P$, Jensen IP, Jeune B, Ebbesen N, Bremmelgoard A, Moller BR. Few microorganisms associated with bacterial vaginosis may constitute the phatologic core:A population-based microbiologic study among 3 596 pregnant women. Am J O bstet Gynecol 1998;178(3):580-587. 3. Priestley CJ, Kinghorn GR. Bacterial vaginosis. Br J Clin 1996;50(6):331-334.

4. Perera JT, Clayton Y.The incidence species distribution and antifungal sensitivity pattern in Sri Lankan women. Mycoses 1994:37(9-10): 357-360.

5. Mikamo H, Sato Y, Hayasaki y Tamaya T. C urrent status and fluconazol treatment of pelvic fungal gynecological infections. Chemotherapy 2000;46(3):209-212.

6. González-Pedraza A, 0 rtiz-Zaragoza C, Inzunza-Montiel AE, Ponce-Rosas ER. Candidiasis vaginal: diagnóstico y tratamiento en atención clínica primaria. Aten Primaria 1998;21(6):395-398. 7. Kiliç HI,Atan A,A koz I,A kaun I,Alpay E. Microbial assessment of patients with vaginitis, vaginal discharge and urine cultures. Mikrobiyol Bull 1991;25(4):313-320.

8. Iyer SV, D eodhar L, G ogate A. Microbiological evaluation of female patients in STD clinics. Indian J Med Res 1991;93:95-97.

9. Horowitz BJ, Giaquinta D, Ito S. Evolving pathogens in vulvovaginal candidiasis: Implications for patient care. J Clin Pharmacol 1992;32: 248-255.

10. Rivera RL, Q uiterio TM, Cruz VA, Conde G lez CJ. Prevalence of bacterial vaginitis and vaginosis:Association with clinical and laboratory features and treatment. Ginecol 0 bstet Mex 1996;64:26-35.

11. Pliutto AM. Laboratory diagnosis of bacterial vaginosis. Klin Lab Diagn 1997;3:16-18.

12. Lo BB, Philippon M, C unin P, Meynard D, Tandia-Diagana M.The microbial etiology of genital discharges in N ouakchott, Mauritania. Bull Soc Pathol Exot 1997;90(2):81-82.

13. Behets FM, D esormeaux J, Joseph D, Adrien M, C oicou G, Dallabetta $\mathrm{G}$ et al. Control of sexually transmitted diseases in Haiti: Results and implications of a baseline study among pregnant women living in cite Soleil Shantytowns. J Infec Dis 1995;172:761-764.

14. Inserberg H, D 'A mato RF. Indigenous and pathogenic microorganisms in humans. En: Ballo us A, Herman K, Inserberg HD, Shadomy HJ, Ed. Manual of clinical microbiology. $5^{\text {th }}$ edition. W ashington, DC:American Society for Microbiology, 1991:7-9.

15.W arren N G, Shadomy HJ.Yeasts of medical importance. En: Balows A, Hermann K, Insenberg HD, Shadomy HJ, Ed Manual of clinical microbiology. $5^{\text {th }}$ Edition. American Society for Microbiology, 1991:617-618.

16. Ringdahl EN . Treatment of recurrent vulvovaginal candidiasis. Am Fam Physician 2000;61:3306-3317.

17.Tam MT,Yungbluth M, Myles T. Gram Sstain method shows better sensitivity than clinical criteria for detection of bacterial vaginosis in surveillance of pregnant low-income women in a clinical setting. Infec Dis 0 bstet Gynecol 1998;6:204-208. 